\title{
Head roll stabilisation in the nocturnal bull ant Myrmecia pyriformis: implications for visual navigation
}

\author{
Chloé A. Raderschall ${ }^{1}$, Ajay Narendra ${ }^{1,2}$ and Jochen Zeil ${ }^{1, *}$
}

\begin{abstract}
Ant foragers are known to memorise visual scenes that allow them to repeatedly travel along idiosyncratic routes and to return to specific places. Guidance is provided by a comparison between visual memories and current views, which critically depends on how well the attitude of the visual system is controlled. Here we show that nocturnal bull ants stabilise their head to varying degrees against locomotion-induced body roll movements, and this ability decreases as light levels fall. There are always un-compensated head roll oscillations that match the frequency of the stride cycle. Head roll stabilisation involves both visual and non-visual cues as ants compensate for body roll in complete darkness and also respond with head roll movements when confronted with visual pattern oscillations. We show that imperfect head roll control degrades navigation-relevant visual information and discuss ways in which navigating ants may deal with this problem.
\end{abstract}

KEY WORDS: Ants, Navigation, Head stabilisation, Vision, Image-matching, Dim light

\section{INTRODUCTION}

Visual memories can provide navigational guidance only if the movement and the orientation of the visual system are controlled during acquisition and recall. As animals move, they experience complex retinal image shifts (e.g. Eckert and Zeil, 2001; Kress and Egelhaaf, 2014; Schilstra and van Hateren, 1998; Srinivasan and Bernard, 1975). Image motion generated by pure translation provides useful information on heading direction and on the relative distance of objects (Collett et al., 1993), but image motion signals generated by rotation degrade the quality of that visual information (reviewed in Zeil et al., 2008). To minimise rotations of the visual system, animals engage in compensatory eye movements that help stabilise gaze during locomotion (e.g. Hengstenberg, 1993; Land, 1999, 2015; Zeil et al., 2008).

The visual systems of insects, the compound eyes and ocelli, are part of the head capsule and their orientation is controlled by moving the head around a single neck joint (Hengstenberg, 1993). Flying insects are able to keep the roll and pitch orientation of the head constant despite large, but predictable, changes of orientation of the thorax associated with flight control (Beatus et al., 2015; Boeddeker et al., 2010; Goulard et al., 2015; Land, 1973; Schilstra and van Hateren, 1998; Viollet and Zeil, 2013). Walking insects, however, face not only the predictable stride-cycle-dependent body

\footnotetext{
${ }^{1}$ Research School of Biology, The Australian National University, 46 Sullivans Creek Road, Canberra, Australian Capital Territory 2601, Australia. ${ }^{2}$ Department of Biological Sciences, Macquarie University, W19F, 205 Culloden Road, Sydney, New South Wales 2109, Australia

*Author for correspondence ( jochen.zeil@anu.edu.au)
}

Received 1 November 2015; Accepted 24 February 2016 oscillations but also unpredictable ground topography-dependent body rotations (Kress and Egelhaaf, 2012, 2014). In the context of visual navigation, it is thus important to investigate to what extent pedestrian navigators, such as ants, are able to control head orientation around the roll and pitch axes while walking (Ardin et al., 2015 and literature review therein). Ants are assumed to memorise views of the nest environment during learning walks before they head out on their first foraging trip (e.g. Graham et al., 2010; Jayatilaka et al., 2014; Müller and Wehner, 2010; Narendra et al., 2013a) and also along the paths they travel (Baddeley et al., 2012; Collett, 2009; Kohler and Wehner, 2005; Mangan and Webb, 2012; Wehner et al., 1996). To make use of these memories for navigation, insects must be able to hold their visual system in defined orientations during acquisition and recall (e.g. Duelli, 1975; Weihmann and Blickhan, 2009; Ardin et al., 2015).

Here we investigate head roll orientation in the nocturnal ant Myrmecia pyriformis Smith 1858, which has previously been shown to rely heavily on vision for navigation (Narendra et al., 2013c; Reid et al., 2011). We ask (1) whether nocturnal ants exhibit compensatory head movements to stabilise their gaze while walking, (2) to what extent their visual system is involved in head stabilisation, and (3) whether a change in ambient light intensity affects head stabilisation. To the best of our knowledge, this is the first attempt to investigate head roll stabilisation in a walking insect in its natural visual environment outdoors. We achieved this by studying ants that walked on a twisted packing band that was fastened to an optical bench (Fig. 1).

\section{MATERIALS AND METHODS}

\section{Ants and preparation}

Workers of the bull ant, M. pyriformis, were caught during the evening twilight at the base of their nest-specific foraging tree at the Australian National University campus field station, Canberra $\left(35^{\circ} 16^{\prime} 50.14^{\prime \prime} \mathrm{S}\right.$, $\left.149^{\circ} 06^{\prime} 42.13^{\prime \prime} \mathrm{E}\right)$. They were transferred to the laboratory inside foamstoppered vials and provided with sugar solution. The following day, ants were immobilised on ice and a small flag (paper strip) was glued onto their mesosoma using super glue (UHU, Bühl, Germany) to facilitate monitoring of body roll (Fig. 2A). Upon preparation, ants were given at least $1 \mathrm{~h}$ resting time to recover from cooling and handling before experiments began. A second group of ants was prepared for tethering to test for an optomotor response. These ants were attached to a small wire using dental glue (SDI, shade modification, Bayswater, VIC, Australia), which allowed us to tether the ants inside a horizontally oriented rotating drum (for details, see below and Fig. 6A). The ants were also given a small paper ball to hold with their legs, which they rotated as they attempted to walk. The paper ball was necessary for the ants to keep calm and behave naturally.

\section{Experimental setup for freely walking ants}

To analyse the extent to which ants stabilise their head while walking freely, we took advantage of the ants' preference to walk 

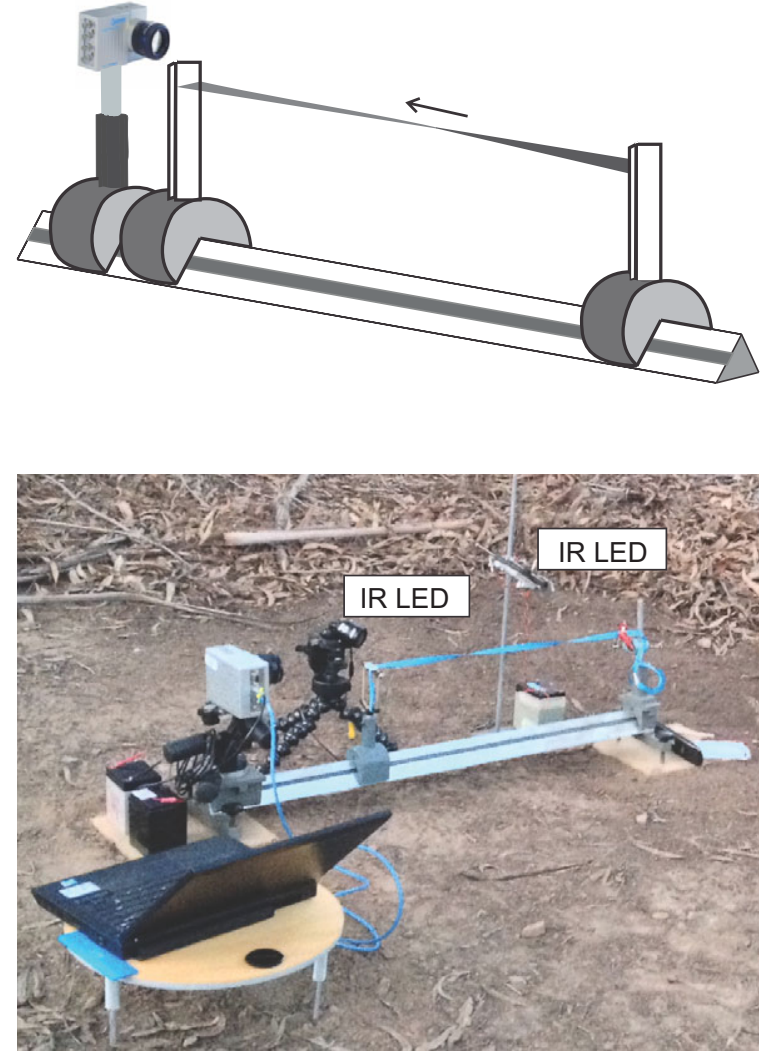

Fig. 1. Experimental setup with twisted walking band. Ants walked over a twisted band that was held tight between two poles mounted on an optical bench. Ants were filmed from the front with a high-speed camera under infrared illumination. Arrow indicates the ants' direction of travel. Photograph shows setup in the field, including locations of infrared illumination.

along sticks. Ants were placed on a twisted plastic packing band (1 $\mathrm{cm}$ wide) that ran $30 \mathrm{~cm}$ above the ground between two aluminium rods fixed to an optical bench of $1 \mathrm{~m}$ in length (Fig. 1). Using the same portable setup, we filmed ants both outdoors under ambient light conditions (October 2013-February 2014), and in a typically cluttered indoor laboratory environment (December 2013) under normal room light and in complete darkness. Ants were individually released on the band throughout the evening astronomical twilight period. We frontally filmed ants walking over a stretch of approximately $10 \mathrm{~cm}$ of the twisted band using a high-speed digital camera (CR600×2, Optronis, Kehl, Germany) mounted on the optical bench, running at 125 frames s ${ }^{-1}$. Images $(1280 \times 1024$ pixels $)$ were saved to a DELL portable computer running Optronis TimeBench software and later converted to uncompressed 8-bit jpeg files for further processing. The setup was illuminated with two infrared LED panels with a peak wavelength of $850 \mathrm{~nm}$, which the ants cannot perceive (Ogawa et al., 2015). We measured light intensities for both the indoor (dark: $0.00 \mathrm{~W} \mathrm{~cm}^{-2}$; room light: $5.26 \times 10^{-6} \mathrm{~W} \mathrm{~cm}^{-2}$ ) and outdoor experiments using a radiometer ILT1700 with a SED100/F/L30 detector for increased sensitivity (Warsash Scientific, Redfern, Australia).

\section{Analysis of panoramic images}

We were interested in assessing how serious the effect of imperfect head roll stabilisation is for visual homing. To do this, we assumed that $M$. pyriformis employ some kind of image-matching strategy to localise their nest and to move along habitual routes, as has been
A

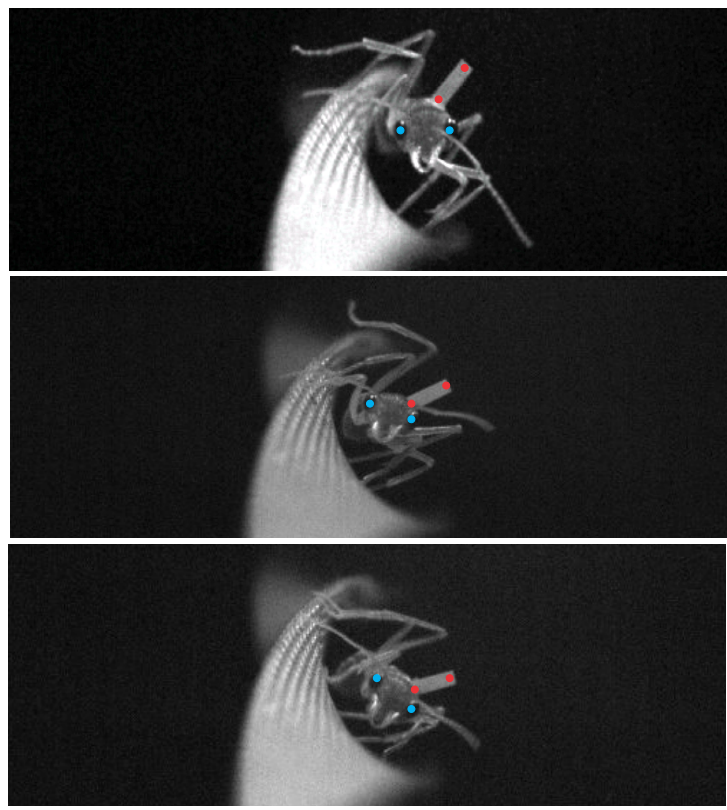

B
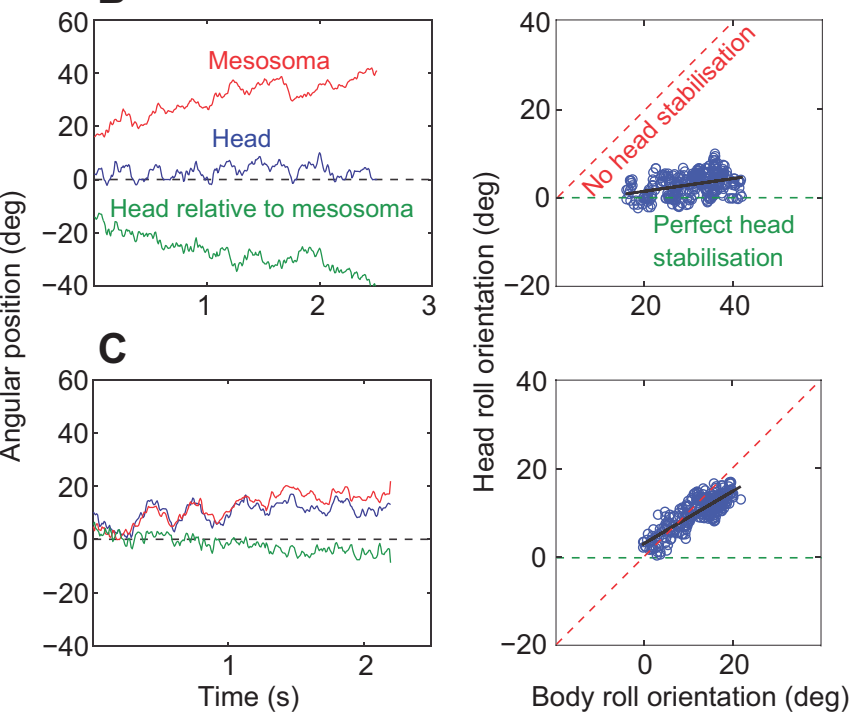

Fig. 2. Head roll stabilisation in Myrmecia pyriformis ants under natural conditions. (A) Image series showing head roll orientation in an ant experiencing increasing body roll (top to bottom). Coloured dots mark the positions of markers on the head (blue) and the flag glued to the mesosoma (red) that were used to determine head and body roll orientations.

$(B, C)$ Examples of an ant that exhibits near-perfect head stabilisation (B) and one that exhibits almost no head stabilisation $(C)$. Left diagrams: time course of orientation of head (blue), mesosoma (red) and head relative to mesosoma (green). Right diagrams: scatter plots of head and body roll orientation (blue circles) for the sequences shown on the left. Regression lines are shown as black lines. Expected relationships for perfect compensation and for the absence of compensation are indicated by dashed lines.

suggested to be the case for other ants and hymenopteran insects in general (reviewed in Collett et al., 2013; Zeil, 2012). One important piece of information that can be gleaned from a comparison between a remembered and a currently experienced view is the heading direction (towards the nest or along a route), indicated by the direction in which image differences are smallest when scanning and comparing the memorised reference image against the current scene - the minimum of the rotational image difference function 


\section{A}
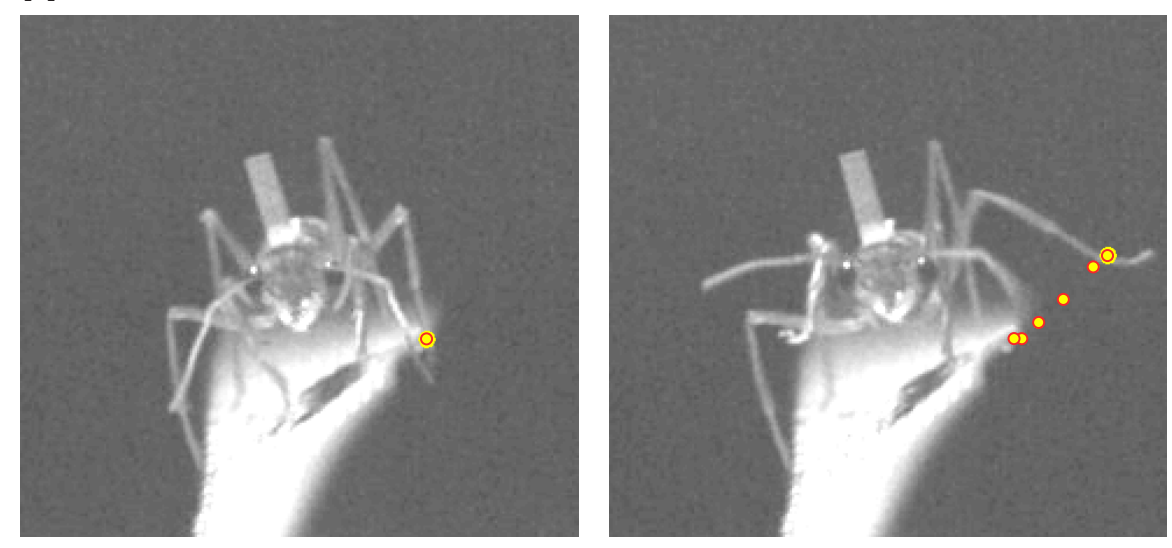

B
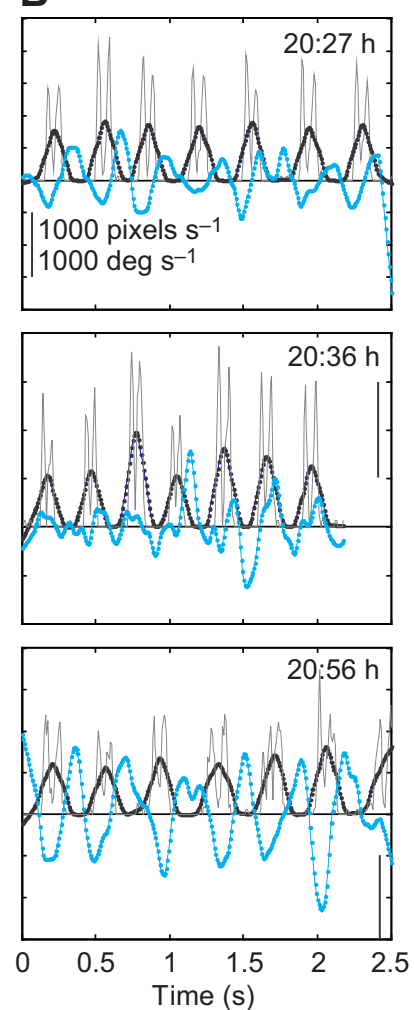
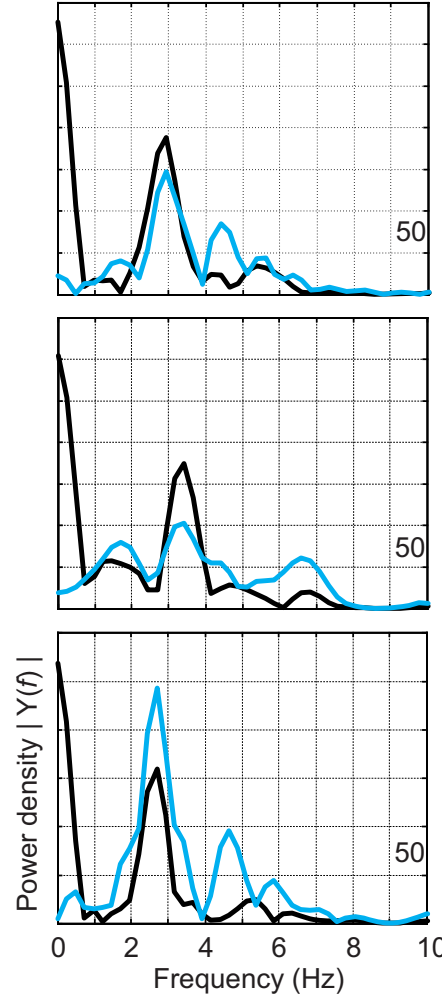
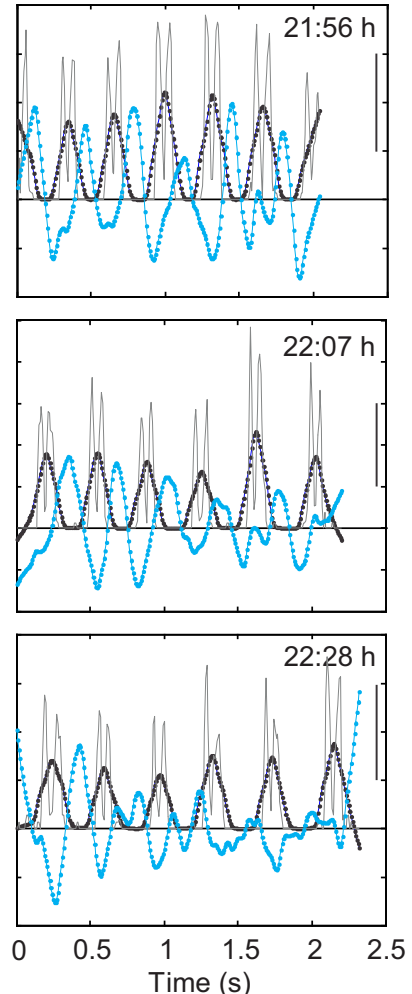
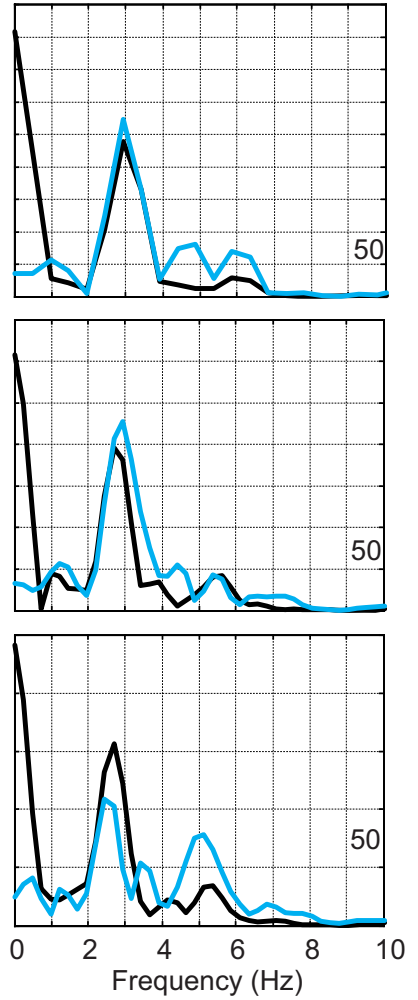

Fig. 3. Stride-cycle-induced head roll oscillations in $\boldsymbol{M}$. pyriformis during walking. (A) Three still images from a video sequence show the left middle leg position for one stride cycle and the $x / y$ coordinates of the proximal tarsus, which were used to determine leg speed. (B) Panels in column 1 and 3 show the time series of head roll velocity [blue; deg s ${ }^{-1}$; smoothed with a 25 -point running average $(200$ ms)] together with the $2 \mathrm{D}$ path velocity of the left middle leg tarsus

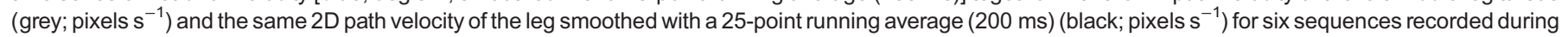
a $2 \mathrm{~h}$ period in the evening (recording times given inside panels). Panels in column 2 and 4 show the power density of leg movements (black) and head roll movements (blue), resulting from a fast Fourier transform of the same sequences. The head roll spectrum has been multiplied by 10 for ease of comparison.

(rotIDF; e.g. Graham et al., 2010; Baddeley et al., 2012; Dewar et al., 2014; Narendra et al., 2013a,b; Stürzl and Zeil, 2007; Zeil et al., 2003). We captured panoramic scenes on a single day at midday using a Sony Bloggie camera (MHS-PM5) at two different locations along the ants' foraging corridor: at the nest and halfway between the nest and the foraging tree (nest-tree distance $12 \mathrm{~m}$ ). The camera was mounted on a Leitz Universal Stage goniometer and levelled using a spirit level before images were captured every $5 \mathrm{deg}$ at roll angles between 0 and $25 \mathrm{deg}$. The circular panoramic images were unwarped to rectangular panoramic images, using a customwritten program (courtesy of W. Stürzl, DLR Germany). The 8-bit greyscale images were converted to floating point arrays and an
$80 \times 80$ pixel Gaussian filter with $\sigma=$ FWHM/2.355 pixels [with full width at half maximum (FWHM) set to twice the interommatidial angle of $3 \mathrm{deg}$ ] was applied to match the average interommatidial angle of the eye of $M$. pyriformis (Narendra et al., 2011). We then compared panoramic view similarities between the two locations along the ants' foraging path by determining the rotIDF (for details, see Stürzl and Zeil, 2007; Zeil et al., 2003). This was done by calculating the pixel differences for 1 pixel shifts between the image at the nest with either itself or with the view halfway along the foraging corridor using the MATLAB circshift function (MathWorks, Natick, MA, USA). The minimum derived by such a comparison corresponds to the highest similarity between the 
A

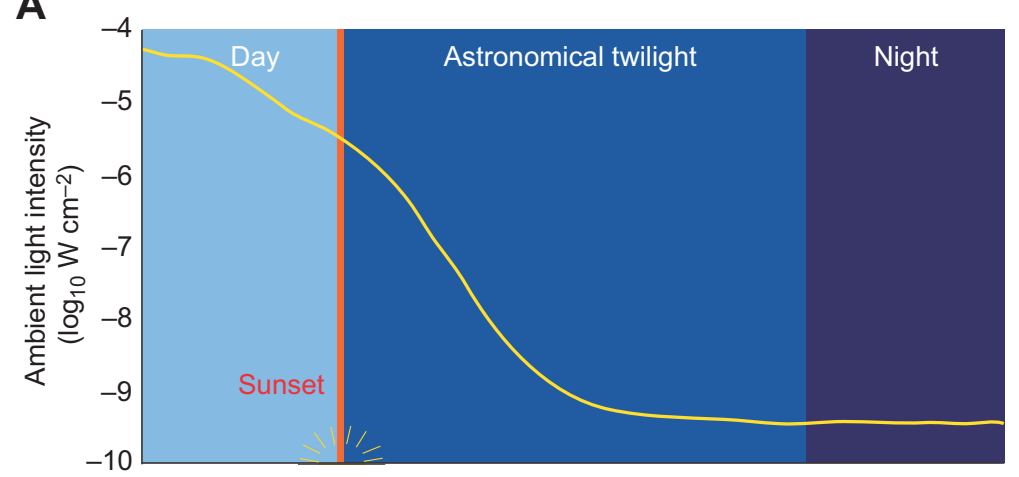

\section{B}

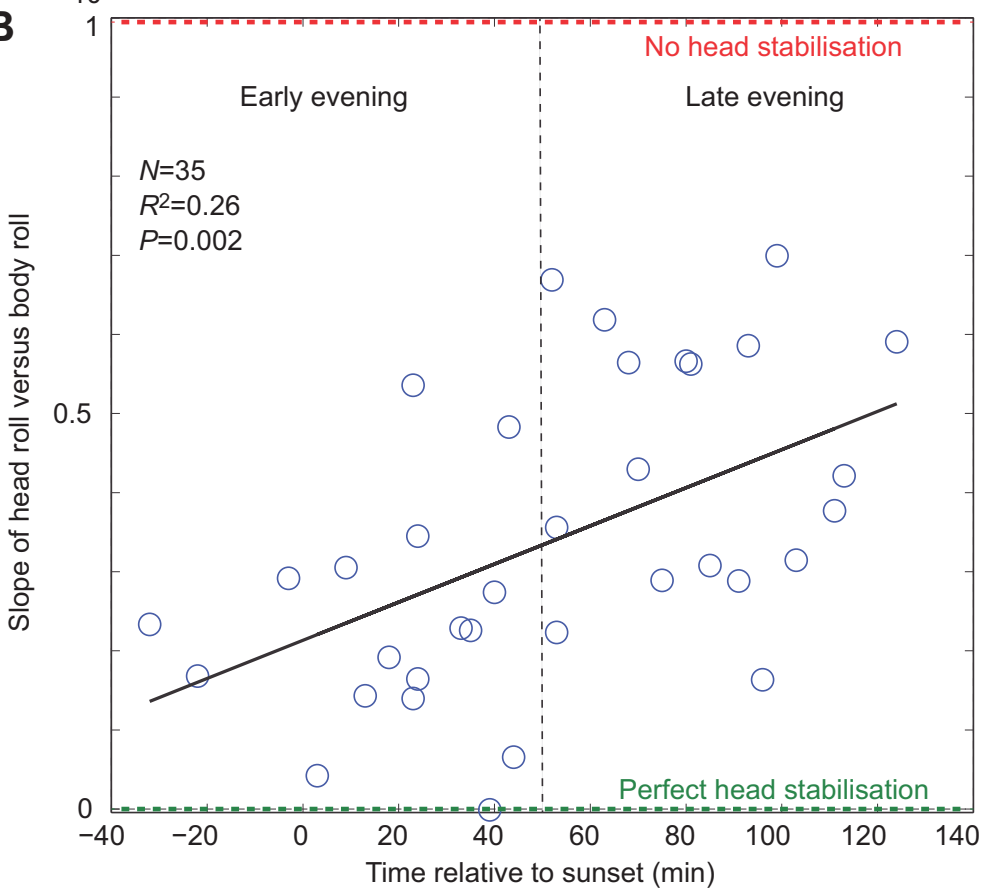

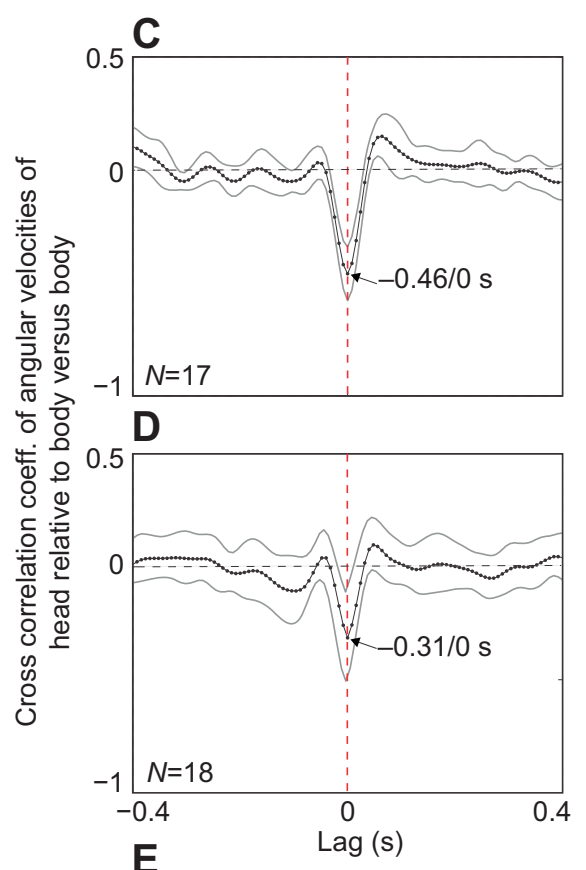

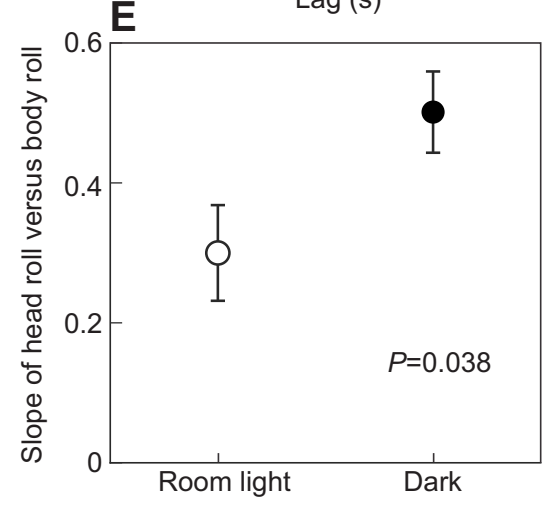

Fig. 4. Head stabilisation in M. pyriformis under ambient light conditions. (A) Ambient light levels (yellow line) on a single day in November 2014 from sunset (vertical orange line) throughout astronomical twilight into night. (B) Values of the slope of head roll orientation versus body roll orientation plotted against time relative to sunset for 35 ants. (C,D) Mean cross-correlation functions of the angular velocity of the head relative to the body and the angular velocity of the body in the early evening $(C)$ and the late evening $(D)$. Black line indicates the mean; grey lines indicate the standard deviation; red dashed line indicates zero lag; arrows point to peak correlation and indicate cross-correlation coefficient/lag. (E) Relationship between the orientation of body roll and head roll of ants tested both under normal room light conditions $(N=14)$ and in complete darkness $(N=20)$. Data are means \pm s.e.m. Data for individual ants, regression lines and slopes are shown in Figs S1 and S2.

views, which tends to coincide with the orientation of homedirected reference views (Baddeley et al., 2012; Narendra et al., 2013a).

\section{Experimental setup for tethered ants}

To identify the contribution of vision for head stabilisation, we tethered ants inside a horizontally oriented rotating cylinder (diameter $14.5 \mathrm{~cm}$, length $14.5 \mathrm{~cm}$ ) that was mounted on an optical bench. The inside of the cylinder carried three different patterns: (1) equally spaced axial black and white stripes of $13 \mathrm{~mm}$ width (spatial frequency of 0.05 cycles deg $^{-1}$ ); (2) a 180 deg black and 180 deg white pattern forming an artificial horizon and (3) a featureless white background. The drum was then manually oscillated around the roll axis back and forth at a frequency of approximately $0.5 \mathrm{~Hz}$ and an amplitude of approximately 15-20 deg, with the aid of a filament line that was wound around the edge of the drum and that ensured a smooth movement of the cylinder. The experiments were performed indoors under normal room light. The inside of the drum was homogeneously illuminated with a ring light guide (Schott Australia, Frenchs Forest, Australia). Frontal views of ants were recorded at 60 frames s ${ }^{-1}$ (image size $1280 \times 1024$ pixels) through the inner opening of the ring light guide with a high-speed digital camera (CR600 $\times 2$, Optronis) that was mounted on the same optical bench as the drum. The camera was equipped with a $90 \mathrm{~mm}$ Panagor macro lens with the aperture set between $\mathrm{f} 11$ and $\mathrm{f} 16$. The camera was connected to a DELL portable computer running Optronis TimeBench software. To monitor the orientation of the cylinder, a thin cardboard strip with two markers was attached to the inside of the drum, which was located in the ants' posterior visual field. Videos were converted to image sequences and stored as uncompressed 8-bit jpeg files for offline processing.

\section{Data analysis}

For freely walking ants, the orientation of the head and the mesosoma was determined by extracting frame-by-frame the $x / y$ 
A
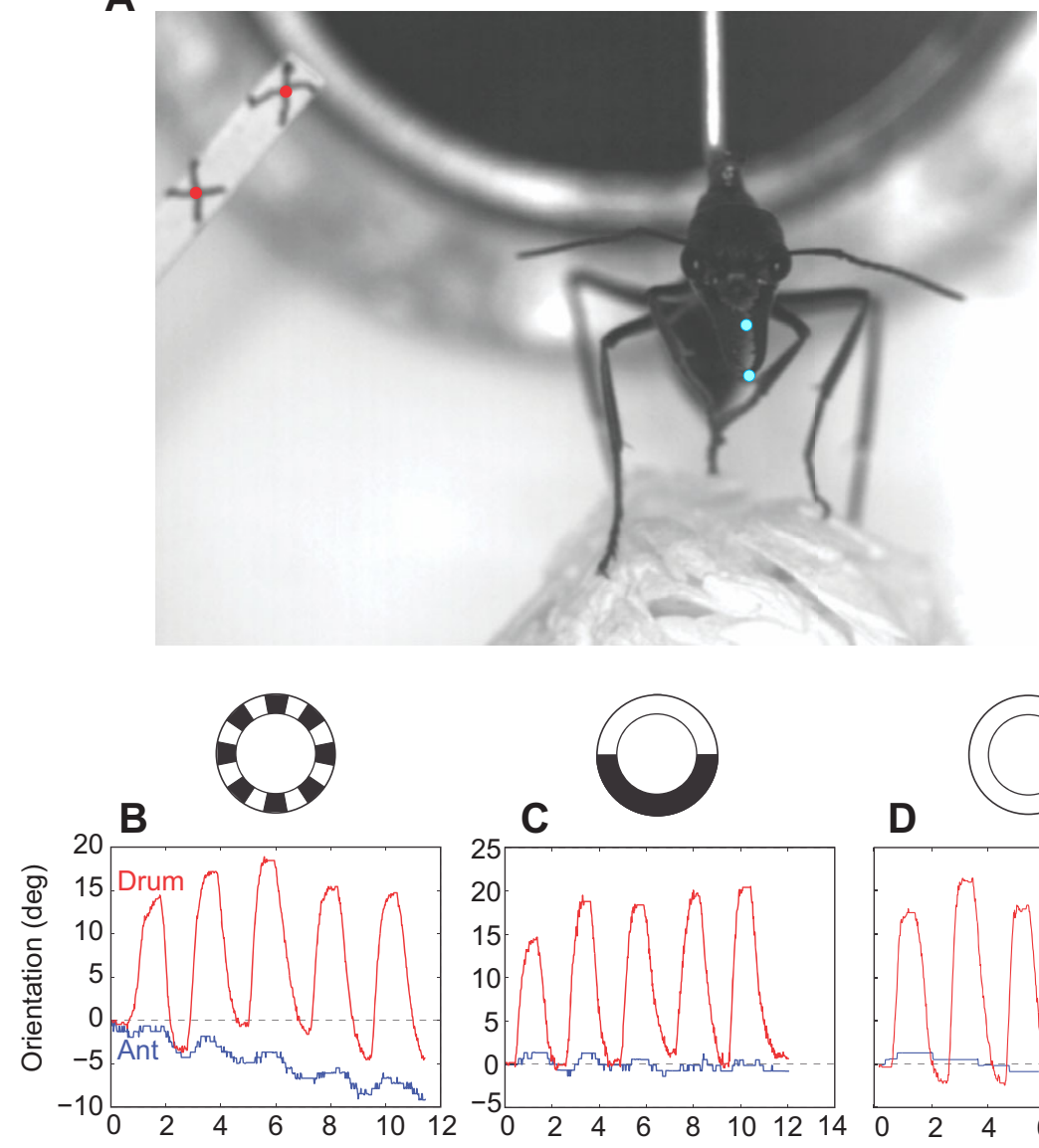

E

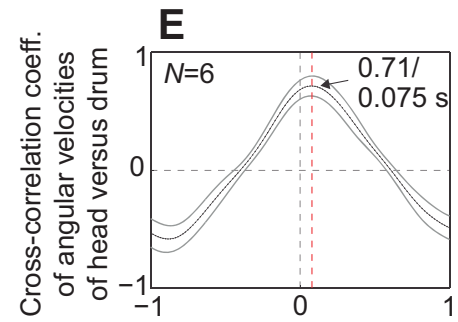

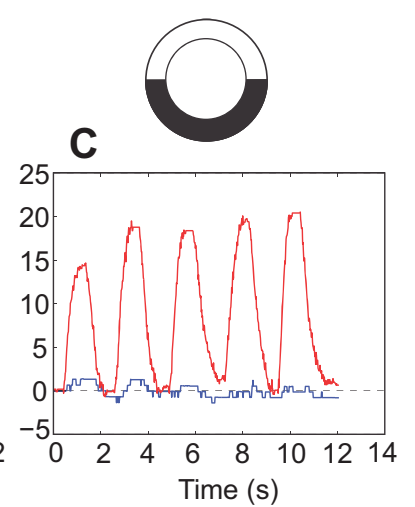
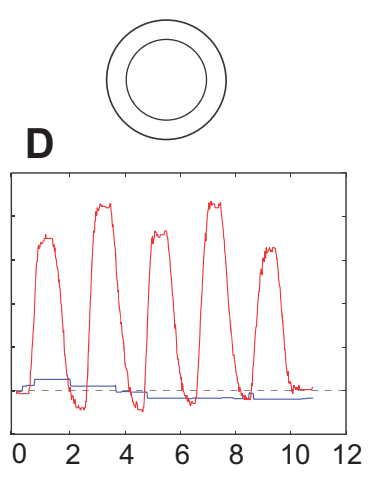

$\mathbf{F}$
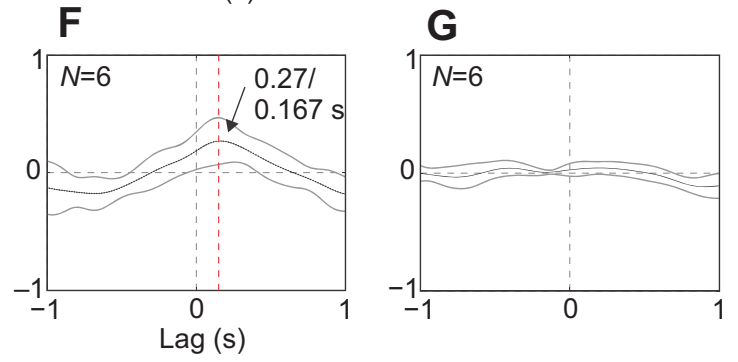

Fig. 5. Head roll stabilisation in M. pyriformis using visual cues alone.

(A) Ants tethered inside an oscillating drum were exposed to $(B, E)$ oscillating stripes,

$(C, F)$ an artificial horizon and $(D, G)$ a blank control. In A, red dots indicate the paper strip used to monitor drum rotation; blue dots indicate the position of the two mandibular teeth used to monitor head orientation. (B-D) Example sequences of head roll orientation (blue) and drum orientation (red) for all three visual conditions. Dashed black line indicates horizontal. $(E-G)$ Mean crosscorrelation functions (black lines) and standard deviations (grey lines) of the angular velocity of the head versus the body for all three visual conditions. Arrows pointing at mean correlation peaks indicate correlation coefficients/lags (red dashed line). Dashed black lines indicate zero lag and zero crosscorrelation coefficient. position of both eyes, and the tip and base of the paper strip mounted on the mesosoma using a custom-written MATLAB program (J. M. Hemmi, R. Parker, The Australian National University) (Fig. 2A). This allowed us to determine whether and to what degree the ants are able to compensate for body roll induced by the twisted band to stabilise their head. For tethered ants, the orientation of the head was determined by digitising the positions of two mandibular teeth. We digitised the $x / y$ position of the tarsus of the left middle leg along with head roll orientation. We illustrate this with six examples (Fig. 3) to show the extent to which head roll movements are generated by the ants' stepping rhythm. To demonstrate that remaining head roll oscillations are stride-cycle induced, we performed a fast Fourier analysis with Hanning window on the time series of the displacement speed of the left middle leg and of the angular velocity of head roll (see Fig. 3). To detect possible delays between body roll and head roll movements, we performed a crosscorrelation analysis between the time series of body and headrelative-to-body angular velocities for each sequence separately, and present the means and standard deviation of the resulting cross- correlation functions in Fig. 4C,D for early and late sequences. Angular velocities were calculated as $V_{t}=\left(\theta_{t+1}-\theta_{t-1}\right) \times f / 2$, where $V_{t}$ is angular velocity at time $t, \theta$ is the orientation of the head or mesosoma and $f$ is the frame rate (frames $\mathrm{s}^{-1}$ ).

\section{RESULTS}

\section{Freely walking ants in their natural habitat}

We captured ants that were leaving the nest and placed them on the twisted plastic packing band (Fig. 1). The ants tolerated band slopes of up to $50 \mathrm{deg}$ before jumping off the band or walking on the edge of the band to avoid larger body roll. Freely walking ants were able to stabilise their head to compensate for body roll under natural light conditions. The ability to do so, however, varied between individuals, and for most ants it was imperfect (least-squares linear regression for the two cases shown in Fig. $2 \mathrm{~B}$ and C, $R^{2}=0.14$, $P<0.01$, and $R^{2}=0.69, P<0.01$, respectively).

Even those ants that on average kept their head horizontal displayed oscillating head roll fluctuations of approximately $5 \mathrm{deg}$ irrespective of the substrate-induced body roll (Fig. 2B). The 
A
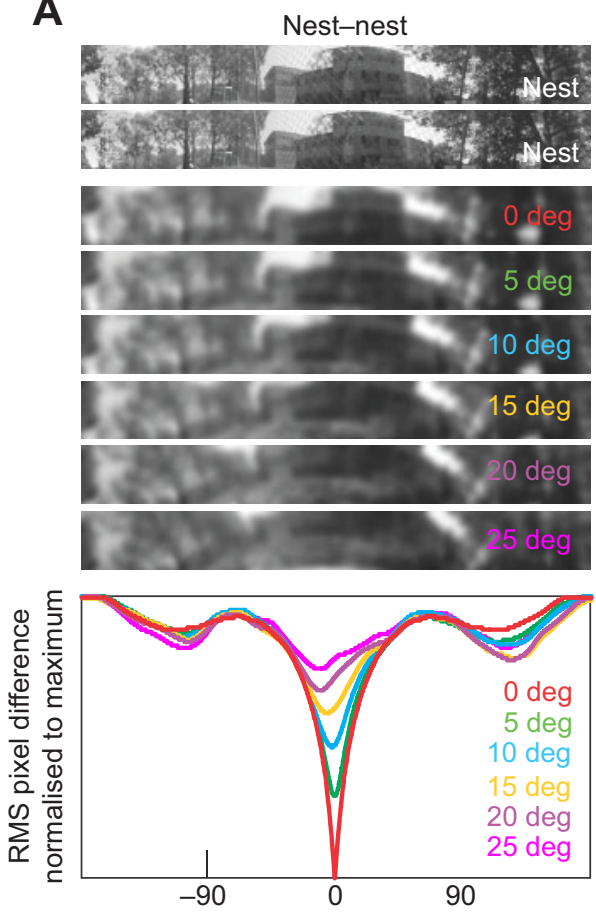

Foraging corridor-nest
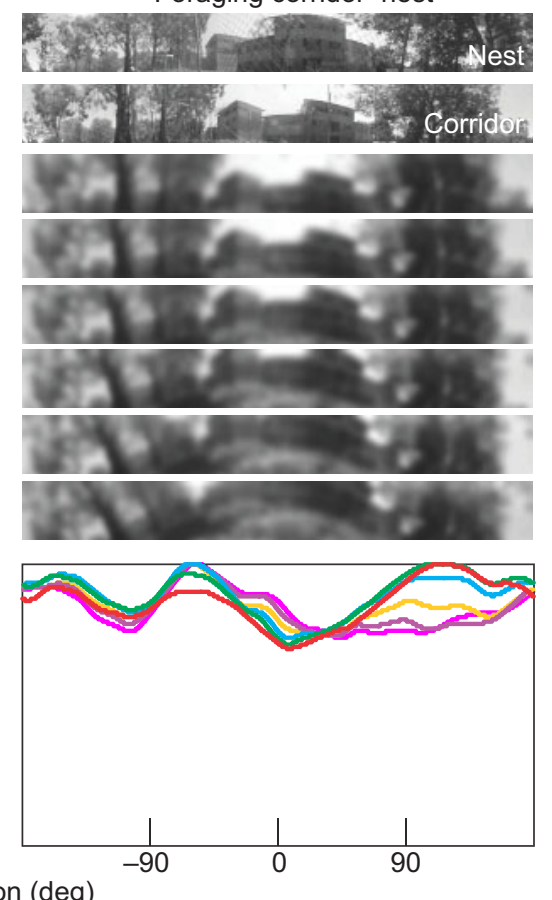

C

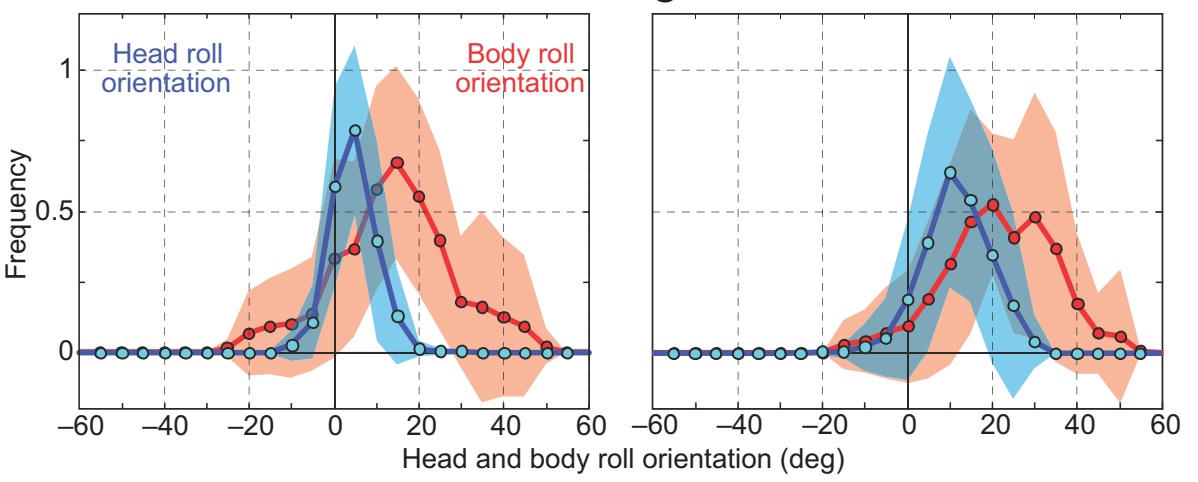

Fig. 6. Head roll control and its implications for panoramic image-matching in $M$. pyriformis. (A) Panoramic images at the nest (left) and along the foraging corridor (right) followed by Gaussian filtered images at different degrees of roll, as indicated by colour-coded numbers. Bottom graphs show correspondingly coloured rotational image difference functions (rotIDF) between the nest image and itself (left) and the nest image and the image halfway along the foraging corridor (right). (B,C) Histograms (mean \pm s.d.) of head (blue) and body roll orientation (red) relative to the horizontal during the early evening $(B)$ and late evening $(\mathrm{C})$. frequency of these remaining head roll oscillations was approximately $3 \mathrm{~Hz}$ and matched the frequency of the stride cycle (Fig. 3). Interestingly, walking speed on the twisted band (as indicated by the stride cycle frequency) did not vary consistently with falling light levels, as shown qualitatively by the six example sequences in Fig. 3, which were recorded over a $2 \mathrm{~h}$ period between 20:27 and 22:28 h.

Typically, workers of $M$. pyriformis begin activity during the evening twilight (Narendra et al., 2010). Light levels drop dramatically during this period and therefore ants experience a wide range of ambient light intensities during their foraging trips (Fig. 4A). Overall, we found that during their natural foraging times, ants were able to compensate for between $30 \%$ and $100 \%$ of the induced body roll (Fig. 4B). Their ability to do so decreased later in the evening (least-squares linear regression, $R^{2}=0.26, P=0.002, N=35$; Fig. 4B), suggesting that head roll stabilisation is to some extent mediated by vision. Unusually for a visual response, both in the early and late evening, ants compensated for the induced body roll with no detectable response lag at $8 \mathrm{~ms}$ temporal resolution (Fig. 4C,D).

We used the same setup to test the ants' ability to stabilise their head in the laboratory in complete darkness as well as under normal room light as a control. In complete darkness, ants were significantly worse at stabilising their head compared with the same individuals tested under normal room light conditions (twotailed $t$-test, $P=0.038$, room light: $N=14$; darkness: $N=20$; Fig. 4E). The order of dark and room-light treatment was chosen randomly, with equal numbers of ants experiencing dark first or room-light first. However, even in complete darkness, ants on average were still able to compensate for $50 \%$ (individuals with a head/body compensation factor of 0.5 ) of the band-induced body roll. Thus, while vision contributes to head roll stabilisation in M. pyriformis workers, there are also non-visual mechanisms, most likely mechanoreceptors (e.g. Goulard et al., 2015) or an efference copy (e.g. Viollet and Zeil, 2013), that mediate this response.

\section{Purely visually controlled head roll stabilisation}

In the absence of information from mechanoreceptors, tethered ants responded differentially to three different visual patterns presented in an oscillating drum (Fig. 5). In response to oscillating horizontal stripes, ants compensated for up to $25 \%$ of the experienced image motion by rotating their head in the same direction (Fig. 5B). When presented with an artificial horizon, ants still responded, although 
the response was much weaker (compensating for approximately $10 \%$ of the drum rotation; Fig. $5 \mathrm{C}$ ). In the absence of visual contrast, ants did not respond to the oscillating drum, confirming that the ants' head roll responses in these experiments were purely visually driven (Fig. 5D).

Ant head movements followed the movement of the stripe pattern with a response lag of $75 \mathrm{~ms}$ (Fig. 5E) and that of the artificial horizon with a lag of $167 \mathrm{~ms}$ (Fig. 5F). Because there was no response to the blank control, there was also no detectable modulation of the cross-correlation function (Fig. 5G).

\section{Implications of imperfect head roll for visual navigation}

Because $M$. pyriformis rely on visual cues for orientation during their foraging excursions, using both the pattern of polarised skylight and the landmark panorama (Narendra et al., 2013c; Reid et al., 2011), we quantified the degree to which imperfect roll stabilisation affects the retrieval of navigational information (specifically heading direction) by determining the rotIDF (see Materials and methods) between a perfectly horizontally aligned reference snapshot and a current scene, which is seen through a visual system that is 'misaligned' by different degrees of roll. Ardin et al. (2015) have recently conducted a similar analysis on the effects of head pitch on navigational information. We find that at the location at which a reference image is taken, the orientation of that image can be recovered even if the current view is rotated up to 20 deg relative to the horizontal (left column, Fig. 6A), as judged by the minima of rotIDFs. However, head roll exceeding $10 \mathrm{deg}$ leads to significant false minima and can altogether abolish a detectable rotIDF minimum when a view at the nest is compared with one along the foraging corridor approximately $6 \mathrm{~m}$ away from the nest (right column, Fig. 6A). In essence, this means that roll and pitch movements of the head limit the range over which panoramic images can provide navigational guidance (e.g. Narendra et al., 2013a; Stürzl et al., 2015; Ardin et al., 2015).

The distribution of residual, un-compensated head roll we measured in our experiments shows that in the early evening the head compensates for approximately $85 \%$ of body roll: the head roll distribution has a median amplitude of 2 deg and a total width of 30 deg (blue histogram, Fig. 6B) against median body roll amplitudes of $13 \mathrm{deg}$ (red histograms). In the late evening the head compensates for approximately 55\%: the head roll distribution has a median amplitude of $9 \mathrm{deg}$ at a total width of 40-50 deg (blue histogram, Fig. 6C) against median body roll amplitudes of $20 \mathrm{deg}$ (red histogram). It is thus unlikely that the ants can continuously access visual cues for navigation while they are walking over uneven ground.

\section{DISCUSSION}

We have shown that walking nocturnal bull ants stabilise their head against body roll, but that there are large variations in how well they achieve this. Part of the variance under natural conditions is due to decreasing ambient light levels, with the ability to stabilise the head decreasing at low light. In addition, remaining head roll movements are synchronised with the ants' stepping rhythm, indicating that ants cannot fully compensate for stride-induced body roll movements. The control of head roll orientation during walking involves both visual and non-visual cues because ants retain some control in absolute darkness. We will first discuss how imperfect head roll compensation affects the use of visual information during navigation and then address some of the control system issues involved in the mechanism of head roll stabilisation.

\section{Implications of head roll orientation for visual navigation}

The importance of head stabilisation has been recognised in the context of the control of locomotion, heading direction, the reduction of image motion blur and the separation of rotational and translational optic flow (Land, 1999, 2015; Zeil et al., 2008; see also Beatus et al., 2015; Goulard et al., 2015). However, it has only recently been considered in the context of the image (snapshot)matching process (Ardin et al., 2015) that is thought to underlie the use of route and place memories for navigation (reviewed in Collett et al., 2013; Zeil, 2012). Recent studies on the Australian jack jumper ant, M. croslandi (Zeil et al., 2014), and on the desert ant Melophorus bagoti (Wystrach et al., 2014) have investigated the way in which ants scan the panorama before deciding where to go, and have concluded that the ants do not look at particular features in the environment, but rather appear to perform a more global matching procedure between current and memorised views (Wystrach et al., 2014; Zeil et al., 2014). Confirming the results obtained by Ardin et al. (2015) for pitch misalignment, we have shown here that misalignments around the roll axis of more than $10 \mathrm{deg}$ between the memorised reference images and the current views are likely to pose serious problems for retrieving navigational information through such global image comparisons. There are a number of ways in which ants may be able to cope with this situation. Ants may be able to restrict acquisition and scanning for visual information to moments in which their head is horizontally aligned, by monitoring the direction of gravity (see below for further discussion). In addition, we know that $M$. pyriformis foragers pause along the route and do so more frequently and for longer durations as ambient light levels drop (Narendra et al., 2013c). Related dayactive ants (Myrmecia croslandi) also repeatedly stop and scan when navigating back to their nest (J.Z., unpublished observations). It is possible that they (and ants in general) restrict image-matching to these stop phases when locomotion-induced blur is minimised and head orientation may be easier to adjust. One testable prediction would be that ants keep their head more horizontally aligned when they perform scanning movements around the yaw axis and/or when they pause along their foraging path (Narendra et al., 2013c), compared with when they are walking (e.g. Wystrach et al., 2014; Zeil et al., 2014). It is also conceivable that there are ways of preprocessing, representing and comparing images that are robust against roll and pitch misalignments, a possibility that remains to be investigated.

Walking bull ants experience recurring head roll of approximately $5 \mathrm{deg}$ at $3 \mathrm{~Hz}$ irrespective of substrate orientation or ambient light levels. This head roll oscillation has the same frequency as the stride cycle and is reminiscent of the yaw head oscillations of approximately $4 \mathrm{deg}$ that were recently described in the walking blowfly Calliphora vicina (Kress and Egelhaaf, 2014). Retinal image shifts that are due to the alternating tripod gait have also been described in predatory tiger beetles Cicindela tranquebarica, who run after their prey using visual guidance and adapt their stride cycle to compensate for the imposed oscillation of their gait when turning towards their prey (Haselsteiner et al., 2014).

\section{Potential sources of information for non-visual head roll control}

Three pieces of evidence indicate that mechanosensory feedforward information may be involved in the compensatory head roll response of M. pyriformis (e.g. Viollet and Zeil, 2013; Goulard et al., 2015): firstly, we did not detect delays at least at a temporal resolution of $8 \mathrm{~ms}$ (Fig. 4C,D); secondly, ants are able to compensate to some degree even in total darkness (Fig. 4E); and 
thirdly, the visual input to head roll orientation is surprisingly weak (Fig. 5B, see below). Potential candidates for mechanosensors are gravity sensors in the mesosoma either in the form of joint-position sensors or joint-load sensors (Mendes et al., 2014). In ants (Mark1, 1962), as in other insects (Apidae: Horn, 1973; Calliphora: Horn and Kessler, 1975; Horn and Lang, 1978; Gryllus: Horn and Bischof, 1983; reviewed in Horn, 1985), hair plates that have been shown to mediate gravity information are found on the antennal joints, the neck, the mesosoma-petiole joint, the petiole-gaster joint and joints between the mesosoma and the coxae. Insects can monitor the deflection of the sensory hairs while they move to precisely determine the relative position of body parts to compute the gravitational force that acts upon them (Mendes et al., 2014). In addition to computing joint position, insects can also use joint load to measure gravity (Hengstenberg, 1993). In insects, specialised stretch receptors (campaniform sensilla) are distributed at strategic points, such as near leg joints on the exoskeleton, and deform when pressure acts upon the cuticula - for example, when the weight distribution on a leg changes during locomotion (Bender and Frye, 2009). Both joint position and joint load could allow ants to monitor the orientation of body parts to detect alignment with the horizontal plane while moving.

\section{The multi-modal head roll control system in ants}

The gain of the visual control loop in tethered M. pyriformis ants is surprisingly low and response latencies are comparatively long for insect visual responses (e.g. Beatus et al., 2015). We note in this context that to the best of our knowledge, there are no studies characterising the optomotor control systems in ants. The observation that delays are longer in response to the horizon pattern compared with the striped pattern has also been described for head roll compensation in wasps (Viollet and Zeil, 2013), and is most probably due to differences in pattern size (Warzecha and Egelhaaf, 2000). With regard to the low gain of the visual control loop, it is important to note that the ants in our optomotor experiments were not walking. One reason for the low gain may therefore be the state- and context-dependent processing of relevant information. For instance, in the blowfly $C$. vicina, a subpopulation of the neck motor neurons (NMN type II) integrates visual information from the compound eyes with mechanosensory information from the halteres (Huston and Krapp, 2009). Visual input only affects the activity of type II NMNs when a non-visual input from the halteres is present concurrently. The fact that these neurons do not produce action potentials in response to visual stimuli alone, but do so when activated in concert with the halteres, stresses the importance of testing head stabilisation in freely moving animals. State- and context-dependent processing may thus explain why head compensation response to visual pattern motion alone in our tethered $M$. pyriformis ants was very weak. However, the visual control loop also does not appear to be sufficient to compensate for stride-cycle-induced roll movements of the body.

Because the ability of $M$. pyriformis to stabilise their head decreases as light levels fall, it will be interesting to now determine whether the optomotor system in congeneric diurnal ants that forage at brighter light intensities contributes more significantly to head roll stabilisation. Most importantly, however, ways need to be found to track the head orientation of ants during their learning walks and over whole foraging journeys, rather than very short sections $(4 \mathrm{~cm}$ : Ardin et al., 2015; $\sim 10 \mathrm{~cm}$ in our analysis). To be able to assess whether a perceived lack of head stabilisation poses serious problems for visual navigation (as discussed here and by Ardin et al., 2015), we would need to know the extent to which head attitude is controlled during the acquisition of visual memories and whether navigating ants compare views intermittently (see Lent et al., 2009) with adjusted head attitude, or continuously, while walking.

\section{Acknowledgements}

We are grateful to Trish Roan for help with digitising video footage and to Frances Jacomb for comments on earlier drafts of the manuscript. We had helpful discussions about data analysis and interpretation with Zoltán Kócsi and Barbara Webb, and technical advice from Wolfgang Stürzl.

\section{Competing interests}

The authors declare no competing or financial interests.

\section{Author contributions}

Identified the problem and designed experiments: C.A.R., A.N. and J.Z.; performed experiments: C.A.R.; data analysis and programming: C.A.R. and J.Z.; wrote initial draft manuscript: C.A.R.; manuscript revision: C.A.R., A.N. and J.Z.

\section{Funding}

We acknowledge funding support from an Australian Postgraduate Award and a Research Supplementary scholarship from the Australian National University to C.A.R., from the Australian Research Council's (ARC) Centre of Excellence Scheme (Centre of Excellence for Vision Science, CEO561903) to J.Z. and from ARC fellowships (DE120100019, FT140100221) to A.N.

Data availability

Data are available on request from the corresponding author.

\section{Supplementary information}

Supplementary information available online at

http://jeb.biologists.org/lookup/suppl/doi:10.1242/jeb.134049/-/DC1

\section{References}

Ardin, P., Mangan, M., Wystrach, A. and Webb, B. (2015). How variation in head pitch could affect image matching algorithms for ant navigation. J. Comp. Physiol. A 201, 585-597.

Baddeley, B., Graham, P., Husbands, P. and Philippides, A. (2012). A model of ant route navigation driven by scene familiarity. PLoS Comput. Biol. 8, e1002336.

Beatus, T., Guckenheimer, J. M. and Cohen, I. (2015). Controlling roll perturbations in fruit flies. J. R. Soc. Interface 12, 20150075.

Bender, J. A. and Frye, M. A. (2009). Invertebrate solutions for sensing gravity. Curr. Biol. 19, R186-R190.

Boeddeker, N., Dittmar, L., Stürzl, W. and Egelhaaf, M. (2010). The fine structure of honeybee head and body yaw movements in a homing task. Proc. R. Soc. $B$ 277, 1899-1906.

Collett, M. (2009). Spatial memories in insects. Curr. Biol. 19, R1103-R1108.

Collett, T., Nalbach, H. O. and Wagner, H. (1993). Visual stabilization in arthropods. In Visual Motion and its Role in the Stabilization of Gaze (ed. F. A. Miles and J. Wallman), pp. 239-263. Amsterdam: Elsevier Science Publishers.

Collett, M., Chittka, L. and Collett, T. S. (2013). Spatial memory in insect navigation. Curr. Biol. 23, R789-R800.

Dewar, A. D., Philippides, A. and Graham, P. (2014). What is the relationship between visual environment and the form of ant learning-walks? An in silico investigation of insect navigation. Adapt. Behav. 22, 163-179.

Duelli, P. (1975). A fovea for e-vector orientation in the eye of Cataglyphis bicolor (Formicidae, Hymenoptera). J. Comp. Physiol. A 102, 43-56.

Eckert, M. P. and Zeil, J. (2001). Towards an ecology of motion vision. In Motion Vision (ed. J. Zanker and J. Zeil), pp. 333-369. Berlin: Springer.

Goulard, R., Julien-Laferriere, A., Fleuriet, J., Vercher, J.-V. and Viollet, S. (2015). Behavioural evidence for a visual and proprioceptive control of head roll in hoverflies (Episyrphus balteatus, Diptera). J. Exp. Biol. 218, 3777-3787.

Graham, P., Philippides, A. and Baddeley, B. (2010). Animal cognition: multimodal interactions in ant learning. Curr. Biol. 20, R639-R640.

Haselsteiner, A. F., Gilbert, C. and Wang, Z. J. (2014). Tiger beetles pursue prey using a proportional control law with a delay of one half-stride. J. R. Soc. Interface. 11, 20140216.

Hengstenberg, R. (1993). Multisensory control in insect oculomotor systems. In Visual Motion and its Role in the Stabilization of Gaze (ed. F. A. Miles and J. Wallman), pp. 285-298. Amsterdam: Elsevier Science Publishers.

Horn, E. (1973). Die Verarbeitung des Schwerereizes bei der Geotaxis der höheren Bienen (Apidae). J. Comp. Physiol. 82, 379-406. 
Horn, E. (1985). Gravity. In Comprehensive Insect Physiology, Biochemistry and Pharmacology, Vol. 6 (ed. G. A. Kerkut and L. J. Gilbert), pp. 557-576. Oxford Pergamon Press.

Horn, E. and Bischof, H.-J. (1983). Gravity reception in crickets: the influence of cereal and antennal afferences on the head position. J. Comp. Physiol. A 150 93-98.

Horn, E. and Kessler, W. (1975). The control of antennae lift movements and its importance on the gravity reception in the walking blowfly, Calliphora erythrocephala. J. Comp. Physiol. A 97, 189-203.

Horn, E. and Lang, H.-G. (1978). Positional head reflexes and the role of the prosternal organ in the walking fly, Calliphora erythrocephala. J. Comp. Physiol. A 126, 137-146.

Huston, S. J. and Krapp, H. G. (2009). Nonlinear integration of visual and haltere inputs in fly neck motor neurons. J. Neurosci. 29, 13097-13105.

Jayatilaka, P., Raderschall, C. A., Narendra, A. and Zeil, J. (2014). Individua foraging patterns of the jack jumper ant Myrmecia croslandi (Hymenoptera: Formicidae), Myrmecol. News 19, 75-83.

Kohler, M. and Wehner, R. (2005). Idiosyncratic route-based memories in deser ants, Melophorus bagoti: how do they interact with path-integration vectors? Neurobiol. Learn. Mem. 83, 1-12.

Kress, D. and Egelhaaf, M. (2012). Head and body stabilization in blowflies walking on differently structured substrates. J. Exp. Biol. 215, 1523-1532.

Kress, D. and Egelhaaf, M. (2014). Gaze characteristics of freely walking blowflies Calliphora vicina in a goal-directed task. J. Exp. Biol. 217, 3209-3220.

Land, M. F. (1973). Head movement of flies during visually guided flight. Nature 243 299-300.

Land, M. F. (1999). Motion and vision: why animals move their eyes. J. Comp Physiol. A Sens. Neural. Behav. Physiol. 185, 341-352.

Land, M. F. (2015). Eye movements of vertebrates and their relation to eye form and function. J. Comp. Physiol. A 201, 195-214.

Lent, D. D., Graham, P. and Collett, T. S. (2009). A motor component to the memories of habitual foraging routes in wood ants? Curr. Biol. 19, 115-121.

Mangan, M. and Webb, B. (2012). Spontaneous formation of multiple routes in individual desert ants (Cataglyphis velox). Behav. Ecol. 23, 944-954.

Markl, H. (1962). Borstenfelder an den Gelenken als Schweresinnesorgane be Ameisen und anderen Hymenopteren. Z. Vergl. Physiol. 45, 475-569.

Mendes, C. S., Rajendren, S. V., Bartos, I., Márka, S. and Mann, R. S. (2014) Kinematic responses to changes in walking orientation and gravitational load in Drosophila melanogaster. PLoS ONE 9, e109204.

Müller, M. and Wehner, R. (2010). Path integration provides a scaffold for landmark learning in desert ants. Curr. Biol. 20, 1368-1371.

Narendra, A., Reid, S. F. and Hemmi, J. M. (2010). The twilight zone: ambient light levels trigger activity in primitive ants. Proc. R. Soc. B Biol. Sci. 277, 1531-1538.

Narendra, A., Reid, S. F., Greiner, B., Peters, R. A., Hemmi, J. M., Ribi, W. A. and Zeil, J. (2011). Caste-specific visual adaptations to distinct daily activity schedules in Australian Myrmecia ants. Proc. R. Soc. B Biol. Sci. 278, 1141-1149.
Narendra, A., Gourmaud, S. and Zeil, J. (2013a). Mapping the navigational knowledge of individually foraging ants, Myrmecia croslandi. Proc. R. Soc. B 280 20130683

Narendra, A., Raderschall, C. A. and Robson, S. K. A. (2013b). Homing abilities of the Australian intertidal ant Polyrhachis sokolova. J. Exp. Biol. 216, 3674-3681.

Narendra, A., Reid, S. F. and Raderschall, C. A. (2013c). Navigational efficiency of nocturnal Myrmecia ants suffers at low light levels. PLOS ONE 8, e58801.

Ogawa, Y., Falkowski, M., Narendra, A., Zeil, J. and Hemmi, J. M. (2015). Three spectrally distinct photoreceptors in diurnal and nocturnal Australian ants. Proc. R. Soc. B. Biol. Sci. 282, 20150673.

Reid, S. F., Narendra, A., Hemmi, J. M. and Zeil, J. (2011). Polarised skylight and the landmark panorama provide night-active bull ants with compass information during route following. J. Exp. Biol. 214, 363-370.

Schilstra, C. and van Hateren, J. H. (1998). Stabilizing gaze in flying blowflies Nature 395, 654-654.

Srinivasan, M. V. and Bernard, G. D. (1975). The effect of motion on visual acuity of the compound eye: a theoretical analysis. Vision Res. 15, 515-525.

Stürzl, W. and Zeil, J. (2007). Depth, contrast and view-based homing in outdoor scenes. Biol. Cybern. 96, 519-531.

Stürzl, W., Grixa, I., Mair, E., Narendra, A. and Zeil, J. (2015). Three-dimensional models of natural environments and the mapping of navigational information. J. Comp. Physiol. A 201, 563-584.

Viollet, S. and Zeil, J. (2013). Feed-forward and visual feedback control of head roll orientation in wasps (Polistes humilis, Vespidae, Hymenoptera). J. Exp. Biol. 216 , 1280-1291.

Warzecha, A.-K. and Egelhaaf, M. (2000). Response latency of a motion sensitive neuron in the fly visual system: dependence on stimulus parameters and physiological conditions. Vision Res. 40, 2973-2983.

Wehner, R., Michel, B. and Antonsen, P. (1996). Visual navigation in insects: coupling of egocentric and geocentric information. J. Exp. Biol. 199, 129-140.

Weihmann, T. and Blickhan, R. (2009). Comparing inclined locomotion in ground-living and a climbing ant species: sagittal plane kinematics. J. Comp. Physiol. A 195, 1011-1020.

Wystrach, A., Philippides, A., Aurejac, A., Cheng, K. and Graham, P. (2014). Visual scanning behaviours and their role in the navigation of the Australian desert ant Melophorus bagoti. J. Comp. Physiol. A. 200, 615-626.

Zeil, J. (2012). Visual homing: an insect perspective. Curr. Opin. Neurobiol. 22 285-293.

Zeil, J., Hofmann, M. I. and Chahl, J. S. (2003). Catchment areas of panoramic snapshots in outdoor scenes. J. Opt. Soc. Am. A 20, 450-469.

Zeil, J., Boeddeker, N. and Hemmi, J. M. (2008). Vision and the organization of behaviour. Curr. Biol. 18, R320-R323.

Zeil, J., Narendra, A. and Stürzl, W. (2014). Looking and homing: how displaced ants decide where to go. Philos. Trans. R. Soc. B Biol. Sci. 369, 20130034. 